\title{
Application of Maxflow/Mincut Algorithm in Phase Unwrapping
}

\author{
Zhen Zhou ${ }^{1}$,Guangmin $\mathrm{Wu}^{1}$,Fanguang Zeng ${ }^{1}$,Jianming Chen ${ }^{1 \mathrm{a} *}$ \\ ${ }^{1}$ Faculty of Science, Kunming University of Science and Technology,Kunming 650500, China \\ ae-mail:584652329@qq.com
}

\begin{abstract}
Phase Unwrapping is an important and difficult problem in interference measurement.Its accuracy directly impacts on the application of the technology. In order to improve the accuracy of unwrapping, we use Bayesian decision theory, establishing the maximum a posteriori Markov random field energy model (MAP-MRF), getting the energy function, so that the phase unwrapping turns into an optimization problem. Then the maximum flow / minimum cut theory is proposed to solve the problem. Experimental results show that the maximum flow / minimum cut theory can well done to optimize the energy function, and the unwrapping results compared to conventional algorithm has an unparalleled advantage on precision.
\end{abstract}

Keywords-information optics; phase unwrapping; Markov random field; maxflow/mincut; discontinuous phase

\section{INTRODUCTION}

In interferometric synthetic aperture radar (InSAR) and magnetic resonance (MR) imaging, because of the reasons of the systems, they can only acquire the phase what was entangled in $(-\pi, \pi)$, the so-called principal phase value or wrapped phase. Phase unwrapping(PU) is the process of recovering the absolute phase from the wrapped phase ${ }^{[1]}$.Phase unwrapping approaches can be subdivided into three different categories : (1)path following methods ${ }^{[2]}$, minimum norm method ${ }^{[3]},(3)$ network programming ${ }^{[4]}$.

Path following algorithms get a appropriate result depends on the assumption that Itoh condition holds along the intergration path. Such as Goldstein branch cut methods easily lead to form the so-called "isolated island" in poor quality of the image area, causing the phase unwrapping failure, at the same time the choice of the branch cut also cannot be the most reasonable ${ }^{[5]}$.

The ideas of minimum norm methods is that find a phase solution for which the $p$ norm of the difference between absolute phase differences and wrapped phase differences is minimized ${ }^{[3]}$. With $p=2$, which is the least square method. At this point we can use FFT or DCT to solve a poisson equation, and get results quickly, but the results are not exact solutions, it is smooth and it will fail when there is a discontinuous phase ${ }^{[6]}$.

This paper uses the method can be attributed to the network programming algorithms. we use Bayesian decision theory, establishing the maximum a posteriori Markov random field energy model (MAP-MRF), getting the energy function where the minimization of it is carried out by maxflow/mincut algorithms.In this paper, two kinds of maxflow/mincut methods are used to optimize the energy function: general augmented path method (Ford-Fulkerson method namely $2 \mathrm{~F}$ method) and improved augmented path method. The experimental results show that compared to the traditional phase unwrapping methods, the method has a great advatange.

\section{MAP-MRF ENERGY FUNCTION}

Let $S=\{(i, j) \mid 1 \leq i \leq N, 1 \leq j \leq M\}$ represent the limited point set what size is $M \times N$, and $X=\left\{x_{s} \mid s \in S\right\}$ represent a random field, $x_{s}$ represent a label field what it's on the random $X$ and the a state space is $k=\{1,2, \cdots, L\}$. The ture phase is $\phi$ and wrapped phase is $\varphi$, and at each pixel are treated as random varibles, and their corresponding images random fields denoted as $\Phi=\left\{\phi_{\mathrm{m}, \mathrm{n}}(\mathrm{m}, \mathrm{n}) \in \mathrm{S}\right\}$, $\psi=\left\{\varphi_{\mathrm{m}, \mathrm{n}}(\mathrm{m}, \mathrm{n}) \in \mathrm{S}\right\}$, and

$$
\psi=\Phi+2 k \pi
$$

So,the phase warapping can be solved as a estimation problem. We use the maximum a posterior estimation (MAP), getting the equation,

$$
\hat{\Phi}=\arg \max p(\Phi \mid \psi)=\arg \max p(\psi \mid \Phi) p(\Phi)
$$

where $p(\Phi \mid \psi)$ and $p(\Phi)$ is the posterior and prior probablities, $p(\psi \mid \Phi)$ is the likelihood function. To solve the MAP problem,we need specify the likelihood function and the prior probability.

(1) Likelihood Function: We define the likelihood function as

$$
P(\psi \mid \Phi)=\delta(\psi-W(\Phi))
$$

where $\delta$ is the Dirac delta function. Givene the maximun likelihood estimate of true phase is not unique. So some constraints conditions are incorporated in the prior probability. 
(2) Prior Probability : We apply a Markov random field to model the "ture" phase images, and use the equivalence of Markov and Gibbs, getting the prior probability

$$
P(\Phi)=\frac{1}{Z} \exp \left(\frac{-U(\Phi)}{T}\right)
$$

where $\mathrm{Z}$ is a probability distribution normalization constants of partition function, $\mathrm{T}$ is a temperature parameter, define as $1 . U(\Phi)$ energy function, and

$$
U(\Phi)=\sum_{c \in C} V_{c}\left(\phi_{c}\right)
$$

Where $V_{c}\left(\phi_{c}\right)$ is the potential function of a clique $c, \mathrm{U}(\Phi)$ is the summation of all the cliques.

According to the neighborhood system, we can know that there are horizontal and vertical directions, therefore,

$$
U(\Phi)=\sum_{c \in C} V_{c}\left(\phi_{c}\right)=\sum_{(i, j) \in S}\left[V\left(\phi_{i, j}, \phi_{i, j-1}\right)+V\left(\phi_{i, j}, \phi_{i-1, j}\right)\right.
$$

where $V\left(\phi_{i, j}, \phi_{i, j-1}\right)=V\left(\phi_{i, j}-\phi_{i, j-1}\right)$,

$$
V\left(\phi_{i, j}, \phi_{i-1, j}\right)=V\left(\phi_{i, j}-\phi_{i-1, j}\right)
$$

then

$$
\begin{aligned}
\hat{\Phi}= & \arg \max _{\Phi}\left\{\prod_{i, j \in S} \delta\left[\varphi_{i, j}-W\left(\phi_{i, j}\right)\right] \times\right. \\
& \exp \left(-\sum_{i, j \in S}\left[V\left(\phi_{i, j}-\phi_{i, j-1}\right)+V\left(\phi_{i, j}-\phi_{i-1, j}\right)\right]\right\}
\end{aligned}
$$

Because that we apply the energy function to phase wrapping and there is a equation(1), namely adhibit currrent $\phi$ into the next iteration as the prior information, so it is not necessary to caculate the likelihood function so we can further reduce the equation(7). Therby, we obtain the exponential of (7), and the overall marks $k$ as

$$
k=\arg \min _{k} \sum_{i, j \in S}\left[V\left(\phi_{i, j}-\phi_{i, j-1}\right)+V\left(\phi_{i, j}-\phi_{i-1, j}\right)\right]
$$

Therefore, the target energy function is

$$
E(k \mid \varphi) \equiv \sum_{\{i, j\} \in S} V\left(\phi_{i, j}-\phi_{i, j-1}\right)+V\left(\phi_{i, j}-\phi_{i-1, j}\right)
$$

where $\phi_{m, n}=\varphi_{m, n}+2 \pi k_{m, n}$
In conclusion, phase wrappping is a process of estimating the $k$ based on the wrapped phase when the equation(9) be mininmized.

The forms of energy function as follows ${ }^{[7]}$

$$
V(x)= \begin{cases}t h^{(0.001-t h)} \cdot x^{2}, & x \leq t h \\ |x|^{0.001}, & x>t h\end{cases}
$$

where th $=2$. The choices of forms of energy functions and parameters depend on the wrapped phase diagram.

\section{OPTIMIZATION OF ENERGY FUNTION}

Before optimizating the energy functions we must structured a graph and we can get a complete graph from paper [8], and according to the construction process of the energy function to the network diagram we can know that solving the minimum energy function is to solve the minimum cut in the network diagram, its calculation method is divided into two main categories [9] : Push Relabel and Augmenting Paths method. This paper mainly studied with the path augmented method.

\section{A. General Augmenting Path Method}

Define The general augmenting path method is also called Ford-Fulkerson method, namely,2F algorithm, it was mentioned by Ford-Fulkerson and applied to caculate network flow in graph, nowadays this method is widely used in financial, communicaion, social management, traffic management, and so on ${ }^{[10]}$

The process of algorithm is divided into three steps,

(1) Labeling, first of all to label the source node as $(0$,inf), the first component ' 0 ' indicates this node is source, and another component 'inf' means that the flow that come from source is infinity. After the source node has been labeled, considering of the nodes that its adjacent but have not been labeled, and labeling the nodes in order of the number. The first component of the nodes indicates that the current node receives the flow which come from corresponding node, and the another component indicates the values of the accepted flow. In the same way to all other nodes, and it's the end of that the sink node have marks and the sink node's the second component isn't zero. After above that have been completed, indicating that the one of augment paths has been found. Now starting with sink node, the first component represents the specific node which constitutes the augmented path.

(2) Augmenting, The augmented value depends on the second component $\alpha$. The principle is that if the first component of a node is negative in the augmented path, then the value of corresponding flow reduces $\alpha$, otherwise, the value pluses $\alpha$.

(3) Repeating the above two steps operation, until the sink node can not be labeled and the algorithm over.

Now,we have completed the application of maxflow/mincut algorithm in phase unwrapping, and the 
results in chapter 3.2 show that the method has a high solution accuary.

\section{B. Improved Augmenting Path Method}

Although the general augmenting path method can get high precision, but the time complexity of the algorithm is too large, the method is limited in actual application, so the method must be improved. The first difference is that the components of labels are three, includes tree, type, and parent, represent that the node belongs to $\mathrm{S}$ tree or $\mathrm{T}$ tree, the type of node (active node, passive node), and the parent node. the second difference is about the augemented path. The path can be found rely on constructing a searching tree. And to improve the efficiency, starting with the source node and sink node at the same time. And because of the three components, the tree can be updated in time and repeated use. The finally, the expermental results have been shown that the improved method have a high accurary as the same as the general augemented path method, but the consumed time is reduced obviously.

The process of algorithm repeats the three main stages,

- growth stage

- augmentation stage

- adoption

\section{EXPERIMENTAL RESULTS AND ANALYSIS}

Fig.4(a) display a phase image $(128 * 128)$ to be unwrapped, they are synthesized from original absolute phase surfaces formed by Gaussian elevation height of 50 units, and the tilting parameters $k$ is 0.05 , cutting radius is 25 pixels, and cutting angle is $\pi / 4$.

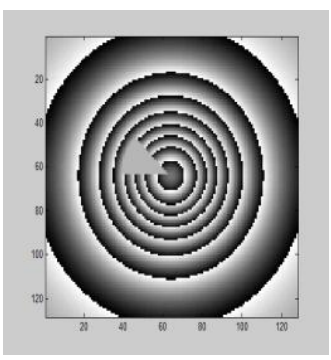

(a)wrapped phase

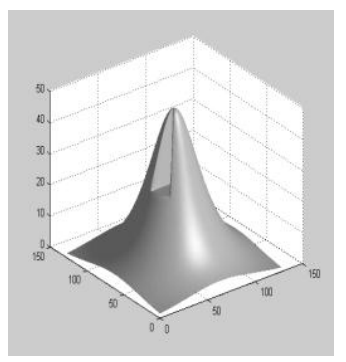

(b)absolute phase
Fig.1. Original data

The results of methods will be evaluated through the rewrapped mean square deviation(RMSD). It is defined as,

$$
\delta=\sqrt{\frac{\sum\left(\varphi(i, j)-\phi^{w}(i, j)\right)^{2}}{M N}}
$$

where $\varphi(\mathrm{i}, \mathrm{j})$ is the original wrapped phase, $\phi^{w}(i, j)$ is the phase what rewrapped the unwrapped phase. $M$ and $N$ represent the size of the images.
A. Results of the Traditional Methods

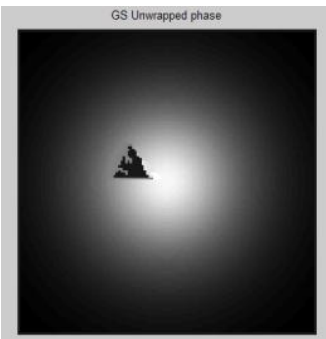

(a) wrapped 2D

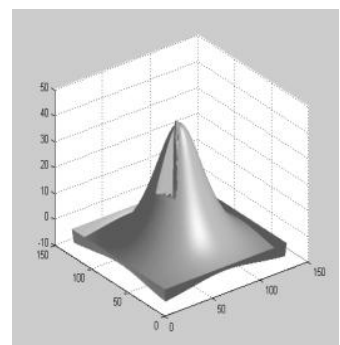

(b) unwrapped 3D
Fig.2. Goldstein branch cut

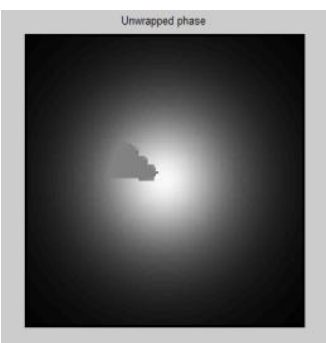

(a) wrapped 2D

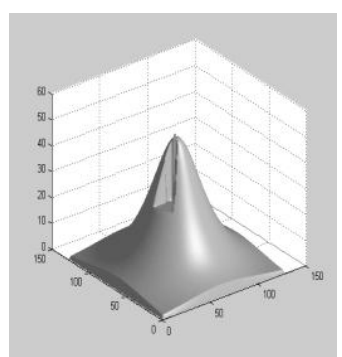

(b) unwrapped 3D
Fig.3. Quality maps guidance

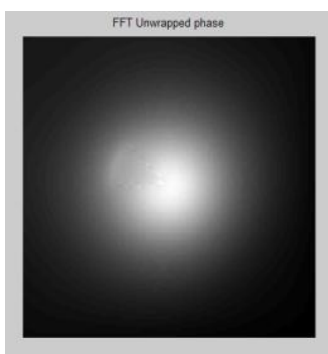

(a) wrapped 2D

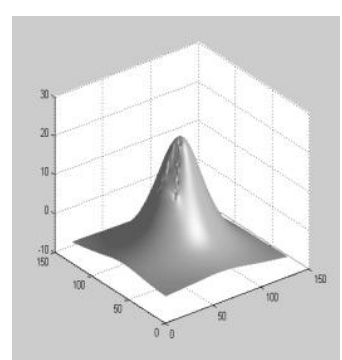

(b) unwrapped 3D
Fig.4. Least square

\section{B. Results of the Maxflow/Mincut Methods}

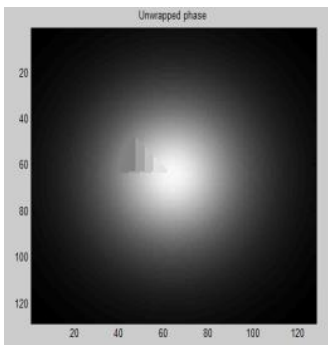

(a) wrapped 2D

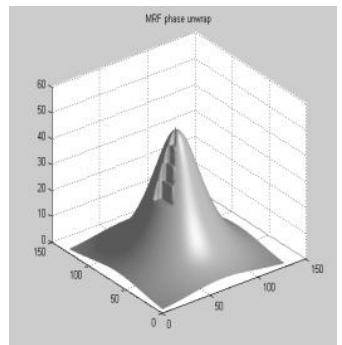

(b) unwrapped 3D Fig.5. General augmenting path

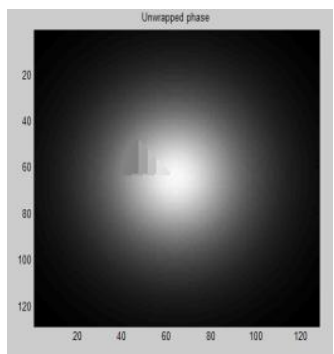


(a) wrapped $2 \mathrm{D}$

(b) unwrapped 3D

Fig.6.Improved augmenting path

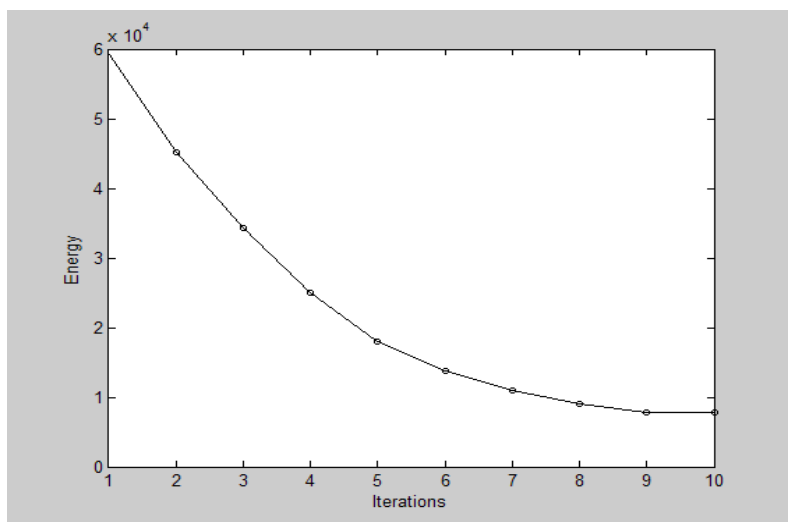

Fig.6. Energy

\begin{tabular}{|c|l|l|}
\hline & $\begin{array}{l}\text { time } \\
(\mathrm{s})\end{array}$ & RMSD \\
\hline Goldstein Branch Cut & 9.48 & 0.4178 \\
\hline Quality Maps Guidance & 22.19 & 0.4052 \\
\hline Least Square & 8.98 & 2.6892 \\
\hline $\begin{array}{c}\text { General Augmenting } \\
\text { Path }\end{array}$ & 18348 & $5.12^{*} 10^{-16}$ \\
\hline $\begin{array}{c}\text { Improved Augmenting } \\
\text { Path }\end{array}$ & 6.55 & $5.12^{*} 10^{-16}$ \\
\hline
\end{tabular}

Table 1. Experimental results

It's obvious that the least square method failed in the discontinuous phase because the method smooths the results, and the Goldstein branch cut method can although get an useful result, it have a poor perform in the terms of accuary compared with the augementing path method, and that the improved augementing path also have a advantage in the terms of time complexity from table 1 .

\section{Conclusion}

1. Phase unwrapping using maxflow/mincut performs well compared with the traditional methods.
2. There are many methods to calculate maxflow/mincut in graph theory, so the direction of phase unwrapping algorithms have been broaden to graph cuts.

3. From the experiments, we can know that the methods well done in the optimizing the energy function, then this methods can also play an effective role in other areas of computer vision.

\section{Acknowledgment}

The corresponding author is Jianming Chen.

\section{REFERENCES}

[1] X Y Li, S Y Mao. Study on phase unwrapping algorithms for interferometricSAR and magentic resonance imaging[J],Chinese Journalof Stereology andImage Ananiysis,2001,6(4):193-197.

[2] K Itoh. Analysis of the phase unwrapping algorithm[J]. Applied Optics, 1982,21(14): 2470-2470.

[3] D J BONE.Fourier fringe analysis:the two-dimensional phase unwrapping problem [J]. Applied Optics, 1991,30(25):3627-3632.

[4] M Costantini. A novel phase unwrapping method based on networkprogramming $[\mathrm{J}]$.Geoscience and Remote Sensing, IEEE Transactionson, 1998, 36(3): 813-821.

[5] F G ZENG, G M WU, J D MAI, J M CHEN.Study on unwrappingof discontinuous phase flaws based on Goldstein branch-cut theory[J]. Laser Technology, 2014, 38(3):335-341.

[6] Z M LIU, J F ZHANG, Y LUO,et al. Experimental and Comparative Study ofInSAR Phase Unwrapping Algorithms[J].Remote Sensing Information, 2012,2:014 : 71-75.

[7] V. Kolmogorov, R. Zabih. What energy functions can be minimized via graph cuts ?[J]. IEEE Transactions on, Pattern Analysis and MachineIntellige-nce, 2004, 26(2): 147-159.

[8] J M Bioucas-Dias, G Valadão. Phase unwrapping via graph cuts[J].Image Processing, IEEE Transactions on, 2007, 16(3): 698-709.

[9] G P WANG, Y WANG, J C REN.Graph theory algorithm theory, implementation and applications[M]. 1st ed. Beijing: Peking University Press. 2011:246- 260.

[10] R BAI. The research of the maximum flow and the minimum cost algorithm[D],Nanjing: Nangjing University ofPosts and Telecommunications, 2012:1-5. 